\title{
Two cases of spontaneous closure of full-thickness macular hole
}

Tan Hun Heng, Kiet Phang Ling, Haslina Mohd Ali

Department of Ophthalmology, Hospital Sultanah Bahiyah, Alor Setar, Kedah, Malaysia

\begin{abstract}
We report two cases of spontaneous closure of full-thickness macular hole (FTMH). The first was in a patient with relieved traction from tractional macular detachment with spontaneous closure after diagnosis at 16 months. The second case is FTMH that developed after vitrectomy from rhegmatogenous retina detachment with spontaneous closure after diagnosis at 9 months. Spontaneous closure of FTMH is rare and the main treatment is vitrectomy.
\end{abstract}

Keywords: full-thickness macular hole, spontaneous closure

\section{Case 1}

A 56-year-old Chinese man with right proliferative diabetic retinopathy and retinitis proliferans with traction affecting the macula developed full-thickness macular hole (FTMH) while he was undergoing panretinal photocoagulation. His visual acuity decreased from $6 / 18$ to $6 / 36$. The macular traction was, however, relieved (captured via optical coherence tomography [OCT]). He was noted to have spontaneous closure of FTMH prior to surgery with visual acuity of $6 / 24$ (Figs. 1 and 2).

\section{Case 2}

A 43-year-old Malay man presented with right macula-off rhegmatogenous retinal detachment. Vitrectomy and gas tamponade were performed, and intraoperatively there was no macular hole $(\mathrm{MH})$. His vision improved from counting fingers to $6 / 36$, but 4 months after surgery there was a drop in vision to $1 / 60$. A FTMH was noted and confirmed via OCT. He refused surgery and 9 months after the diagnosis of FTMH, his vision improved to $6 / 36$ and OCT showed spontaneous closure of FTMH with remnant of subfoveal fluid. Four months later, the amount of fluid further decreased and his vision improved to 6/24.

Correspondence: Tan Hun Heng, Department of Ophthalmology, Hospital Sultanah Bahiyah, KM 6, Jalan Langgar, 05460 Alor Setar, Kedah, Malaysia.

E-mail: hunheng@gmail.com 


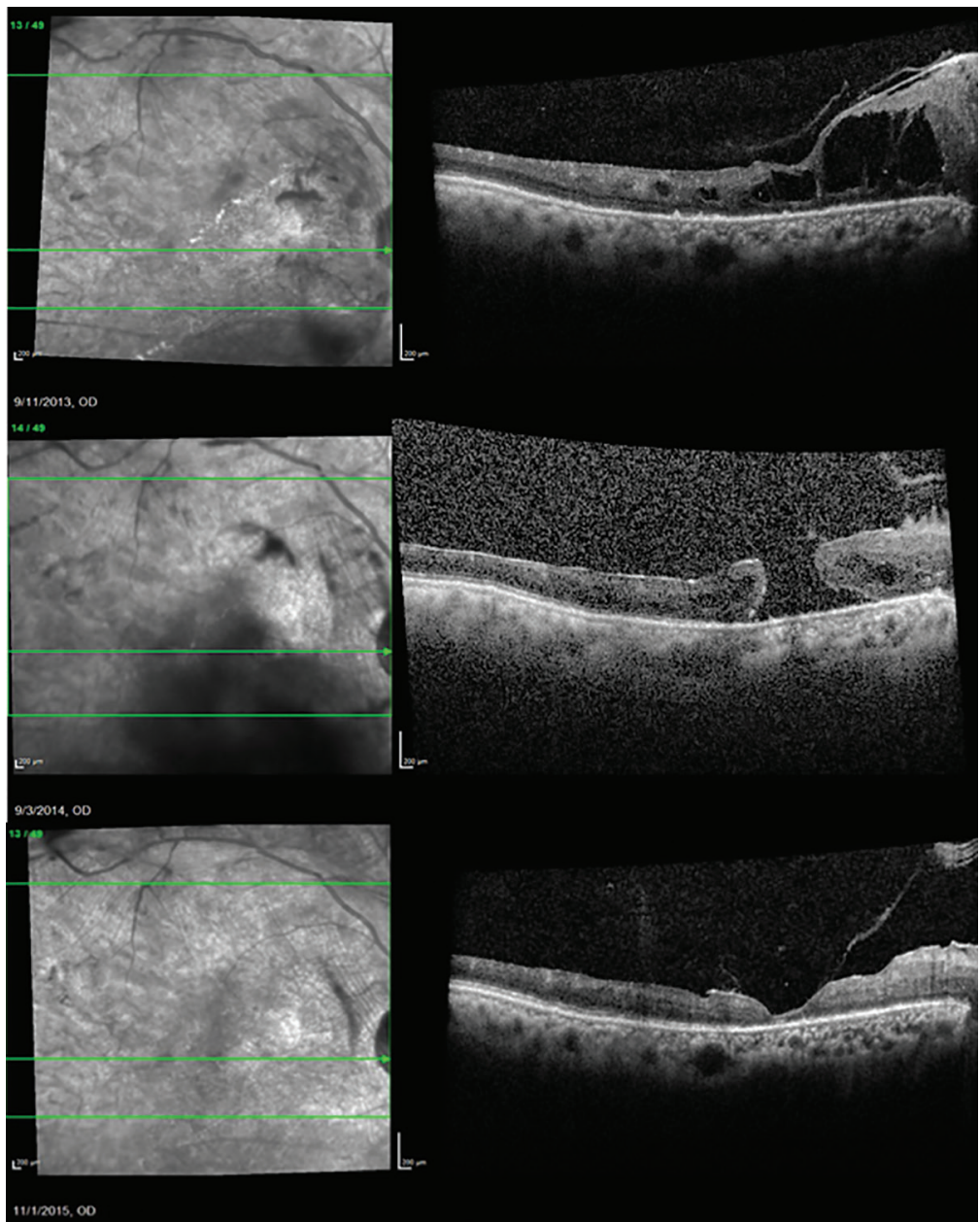

Fig. 1. Serial OCT showing the development of FTMH and subsequent spontaneous closure of FTMH.

\section{Discussion}

FTMH is a retinal break involving the fovea. It is classified as stage 3 based on the modified Gass classification system. ' Spontaneous closure of FTMH is rare, with reported rates between $4 \%$ and $6 \% .^{2}$

There are two major theories that explain the formation of $\mathrm{MH}$ : the anteroposterior vitreofoveal traction and the tangential vitreous traction. ${ }^{1,3}$ The former theory 


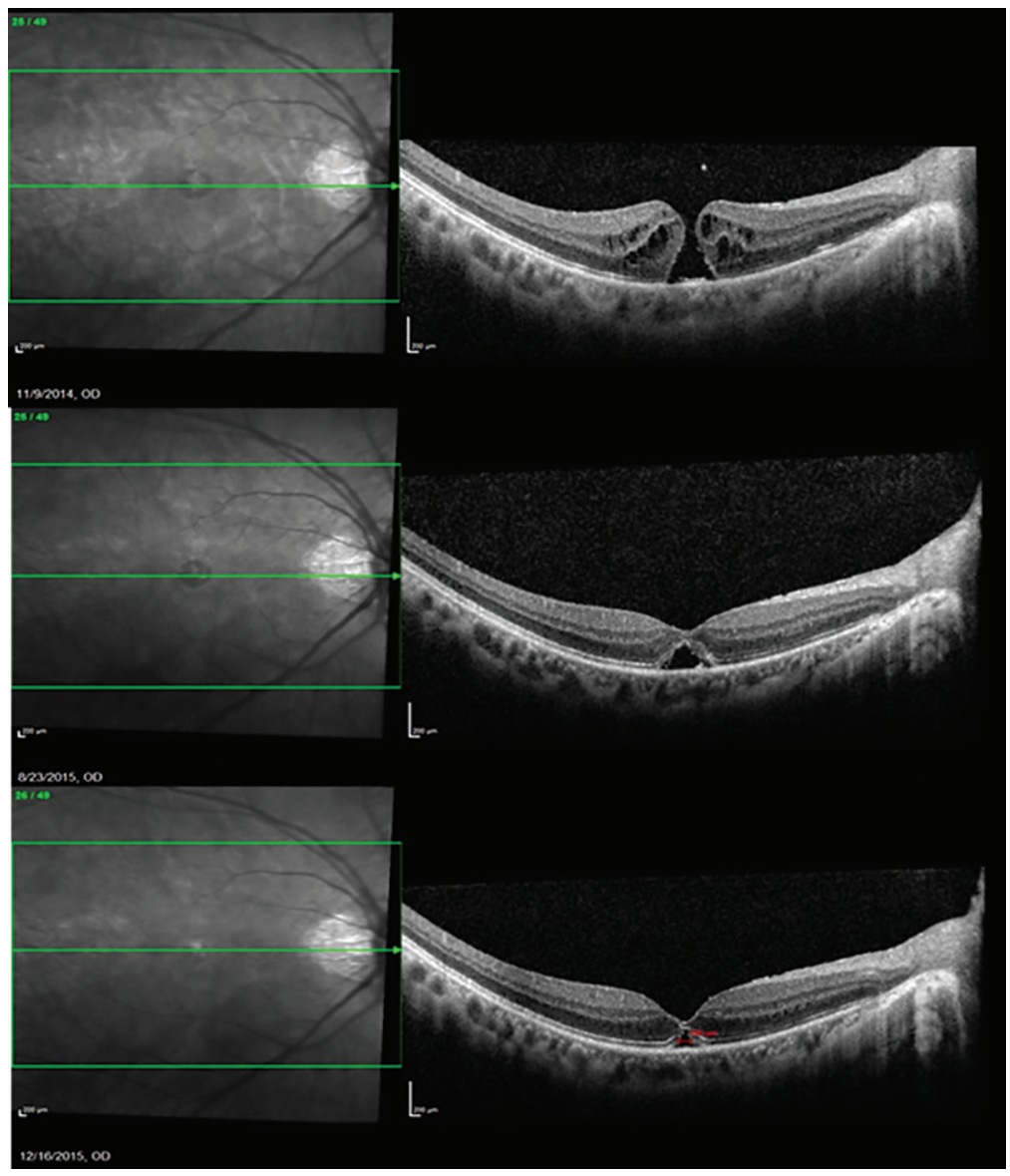

Fig. 2. Serial OCT showing the progression of spontaneous closure of FTMH.

results from a posterior vitreous detachment (PVD) with subsequent foveal cyst formation, whereas the latter is due to condensation and tangential contraction of the prefoveolar vitreous cortex, leading to spontaneous vitreofoveal separation. The mechanism of FTMH formation in both the above-mentioned cases is different. Case 1 developed a FTMH secondary to anteroposterior vitreous traction affecting the macula. However, Case 2 had a FTMH after pars plana vitrectomy, most likely secondary to tangential vitreous traction.

The exact mechanism behind spontaneous closure of FTMH is unclear; however, a few theories have been postulated. Takahashi et al. suggested that the bridging 
of the protruding retinal tissue over the $\mathrm{MH}$ led to closure. ${ }^{4}$ Meanwhile, Suzuki et al. suggested that the process of resolving FTMH could be related to the degeneration of the inner retinal layers due to either atrophy or coalescence of cystoid edema. ${ }^{5}$ Ishida et al. mentioned that a PVD with release of vitreomacular traction plays an important role for spontaneous closure of the $\mathrm{MH}^{6}{ }^{6}$

The current gold standard to diagnose $\mathrm{MH}$ is via $\mathrm{OCT}$, which is widely available. Besides providing an excellent aid in the monitoring of FTMH, it also serves as a guide in the management of the condition. Vitrectomy remains the mainstay of treatment for $\mathrm{MH}$ as it has a favorable outcome, with improvement of visual function in addition to a low recurrence rate.

\section{References}

1. Gass JD. Reappraisal of biomicroscopic classification of stages of development of a macular hole. Am J Ophthalmol. 1995;119(6):752-759. doi:10.1016/S0002-9394(14)72781-3.

2. Inoue M, Arakawa A, Yamane S, Watanabe Y, Kadonosono K. Long-term outcome of macular microstructure assessed by optical coherence tomography in eyes with spontaneous resolution of macular hole. Am J Ophthalmol. 2012;153(4):687-691. doi:10.1016/j.ajo.2011.09.017.

3. Johnson MW, Van Newkirk MR, Meyer KA. Perifoveal vitreous detachment is the primary pathogenic event in idiopathic macular hole formation. Arch Ophthalmol. 2001;119:215-222.

4. Takahashi A, Yoshida A, Nagaoka T, et al. Idiopathic full-thickness macular holes and the vitreomacular interface: a high-resolution spectral-domain optical coherence tomography study. Am J Ophthalmol. 2012;154(5):881-892. doi:10.1016/j.ajo.2012.05.024.

5. Suzuki ACF, Zacharias LC, Nóbrega MJ, Pretti RC, Takahashi WY. Spontaneous closure of macular hole after pars plana vitrectomy: report of two cases. Rev Bras Oftalmol [online]. 2014;73(6):389-391. doi:10.5935/0034-7280.20140080.

6. Ishida M, Takeuchi S, Okisaka S. Optical coherence tomography images of idiopathic macular holes with spontaneous closure. Retina. 2004;24:625-628. http://journals.lww.com/retinajournal/ Fulltext/2004/08000/Spontaneous_Resolution_of_Small_Stage_3_and_4.24.aspx 